\title{
Barriers to Endoscopic Ultrasonography (EUS) in South and South East Asia
}

\author{
Furqaan Ahmed ${ }^{1 *}$ and Khawar Mehdi ${ }^{2}$ \\ ${ }^{1}$ South City Hospital, Karachi, Pakistan \\ ${ }^{2}$ Getz Pharma (Pvt.) Ltd, Pakistan
}

Received: March 02, 2015; Accepted: May 11, 2015; Published: May 20, 2015

*Corresponding author: Furqaan Ahmed, South City Hospital, 128C KDA Scheme \#1, Karachi, Pakistan, 75350, E-mail: furqaan@hotmail.com

\begin{abstract}
Introduction: Endoscopic ultrasonography (EUS) examination has a well defined role in the evaluation of pancreatic diseases, the evaluation of mediastinal lymphadenopathy and masses, and the staging of many gastrointestinal malignancies in the West. EUS FNA is also the standard procedure for sampling pancreatic masses, lymph nodes, and subepithelial lesions.
\end{abstract}

Objective: To assess EUS knowledge and usage among gastroenterologists in South and South East Asia.

Methods: Gastroenterologists from South and South East Asia attending the International Gastroenterology and Hepatology Forum (IGHF) in Yangon, Myanmar in November 2012 were surveyed, using a questionnaire, regarding EUS usage in their countries and their knowledge of the indications for performing an EUS.

Results: 74 Gastroenterologists were included in this study. 69\% (51) were male. $65 \%$ (48) were in academic practice. The doctors were from the following countries: Pakistan 23 (31\%), Sri Lanka 2 (3\%), Myanmar $32(43 \%)$, Philippines 9 (12\%), Laos 5 (7\%), and Cambodia 2 (3\%). The doctors have been in practice for a time period ranging from 1 to 48 years. EUS is available in Pakistan, Philippines, and Sri Lanka and is not available in Myanmar, Cambodia, and Laos. Of the 34 gastroenterologists from countries where EUS is available, only $12(35 \%)$ had EUS facilities in the city where they practice. Two doctors performed EUS themselves. 57 (77\%) said EUS was useful for tumor staging. When asked about which tumors could be staged by EUS, the following were cited: Esophagus $86 \%$ (49), small bowel $40 \%$ (23), rectum $65 \%$ (37), lung $18 \%$ (10), spleen $5 \%$ (3), pancreas $54 \%$ (31), and kidney 2\% (1). When asked about other indications for performing an EUS, the following were cited: 1 . Abnormalities of the gastrointestinal wall $70 \%$ (29), 2. Abnormalities of the kidneys $8 \%$ (3), 3. Abnormalities of the biliary tree $43 \%$ (17), 4. Abnormalities of the heart 8\% (3), 5. Pancreatic cysts 55\% (22), 6. Kidney cysts 8\% (3), 7. Tissue sampling of gastrointestinal wall lesions $48 \%$ (19). When asked about obstacles for performing EUS in their countries, the following reasons were cited: 1 . Cost $50 \%$ (37), 2. Doctor awareness $19 \%$ (14), 3. Patient awareness $18 \%$ (13), 4 . Availability $54 \%$ (40), 5. Lack of trained doctors to perform EUS 49\% (36).

Conclusion: Based on this sample of gastroenterologists representing six countries from the region, there appears to be limited knowledge of the indications for EUS in South and South East Asia. EUS is not available in half of these countries. Issues relating to cost, lack of awareness, availability, and physician training limit EUS use in this region.

Keywords: Endoscopic Ultrasound; Pakistan

\section{Introduction}

Endoscopic ultrasonography(EUS) examination has a well defined role in the evaluation of pancreatic diseases, mediastinal lymphadenopathy and masses, and gastrointestinal malignancies in the West [1]. EUS FNA is also the standard procedure for sampling pancreatic masses, lymph nodes, and subepithelial lesions. The spread of EUS in the West has been facilitated by access to EUS equipment, the development of training programs both within and separate from traditional gastroenterology fellowship training, and by the promotion of the technology and outcome research by endosonographers and gastrointestinal societies.

Previous studies of EUS practice have evaluated training patterns, EUS practice, and knowledge regarding EUS among gastroenterologists internationally. However, in previous surveys of international EUS practice and knowledge, South and South East Asia, have been underrepresented [2-4]. Furthermore, it is not currently known how widely available EUS technology and services are in this region. The objective of this study is to assess EUS knowledge and usage among gastroenterologists in South and South East Asia.

\section{Methods}

This study was conducted by surveying Gastroenterologists from South and South East Asia attending the International Gastroenterology and Hepatology Forum (IGHF) in Yangon, Myanmar in November 2012. The IGHF is a forum created to bring together Gastroenterology and Hepatology faculty, practitioners, and trainees from different countries in the region on a single platform to exchange ideas, learn from one another, and build ties in order to improve the state of gastrointestinal and liver healthcare and research in their respective countries.

The Gastroenterologists were surveyed, using a questionnaire, regarding EUS availability and usage in their countries and their own knowledge of the indications for performing an EUS. Fellows currently in training were excluded.

Using the questionnaire, demographic and practice date were collected including age, gender, type of practice setting, number of years in practice, and the ability to perform EUS. 
Questions were asked about knowledge of EUS indications for different parts of the digestive tract and about tumor staging for different intra-abdominal organs and for lung cancer. Finally, the gastroenterologists were questioned regarding the perceived barriers to the establishment of EUS in their respective countries.

The study was approved by our Institutional Review Board.

\section{Results}

74 gastroenterologists completed the study questionnaire. 51 (69\%) were male and 23 (31\%) were female. 48 (65\%) were in academic practice. The doctors had been in practice for a time period ranging from one to 48 years.

The study participants represented the following six countries in South and South East Asia: Pakistan, Sri Lanka, Laos, Cambodia, Myanmar, and Philippines. EUS is available in Pakistan, Philippines and Sri Lanka. EUS is not available in Myanmar, Cambodia, and Laos. Of the 34 gastroenterologists from those countries where EUS is available, only 12 (35\%) had EUS facilities in the city where they practice. Two gastroenterologists performed EUS themselves.

Participants were then asked about the role of EUS in the evaluation of different intra-abdominal and intra-thoracic organs (Table 1). A majority of gastroenterologists identified a role for EUS in the evaluation of pancreatic cysts and gastrointestinal wall abnormalities. Approximately half of the respondents reported a role of EUS In biliary tree abnormalities and for tissue sampling of gastrointestinal wall lesions. A minority indicated inappropriate use of EUS for the evaluation of abnormalities of the kidneys and the heart and for renal cysts. Finally, participants were asked about EUS for the staging of different malignancies (Table 2). The majority appropriately identified indications for EUS for the staging of esophageal, rectal, and pancreatic tumors. A minority indicated a role for EUS in staging of renal and splenic tumors.

The final section of the questionnaire addressed perceived barriers to EUS in their respective countries (Table 3). Approximately half of the participants identified the following three impediments: availability, cost, and lack of trained endosonographers.

\section{Discussion}

In this survey of knowledge of the indications of EUS among

Table 1: Knowledge of EUS Indications.

\begin{tabular}{|l|l|}
\hline Gastrointestinal wall & $70 \%(29)$ \\
\hline Kidneys & $8 \%(3)$ \\
\hline Biliary tree & $43 \%(17)$ \\
\hline Heart & $8 \%(3)$ \\
\hline Pancreatic cysts & $55 \%(22)$ \\
\hline Renal cysts & $8 \%(3)$ \\
\hline $\begin{array}{l}\text { Tissue sampling of } \\
\text { gastrointestinal wall lesions }\end{array}$ & $48 \%(19)$ \\
\hline
\end{tabular}

Table 2: Knowledge of EUS indications for Tumor Staging.

\begin{tabular}{|l|l|}
\hline Esophagus & $88 \%(46)$ \\
\hline Small bowel & $40 \%(23)$ \\
\hline Rectum & $65 \%(37)$ \\
\hline Lung & $18 \%(10)$ \\
\hline Spleen & $5 \%(3)$ \\
\hline Pancreas & $54 \%(31)$ \\
\hline Kidney & $2 \%(1)$ \\
\hline
\end{tabular}

Table 3: Barriers to EUS.

\begin{tabular}{|l|l|}
\hline Cost & $50 \%(37)$ \\
\hline Doctor awareness & $19 \%(14)$ \\
\hline Patient awareness & $18 \%(13)$ \\
\hline Availability & $54 \%(40)$ \\
\hline $\begin{array}{l}\text { Lack of trained doctors to } \\
\text { perform EUS }\end{array}$ & $49 \%(36)$ \\
\hline
\end{tabular}

gastroenterologists in South and South East Asia, significant deficits were identified in knowledge of the general indications for the procedure as well as its role in tumor staging. Most significantly, EUS is not available in half of the countries that were surveyed in this region. Several obstacles to the widespread adaptation of EUS in the region were also identified, including issues relating to equipment availability, procedure cost, and the lack of trained endosonographers.

Three previous surveys have assessed EUS knowledge and practice among gastroenterologists; two were global in scope and one focused on Asia [2-4]. The most recent of theses were published in 2006 [refs]. The representation of South and South East Asia in these studies was extremely limited. In the study by Das et al, five participants (3\%) were from this region [4]. In the study by Yusuf et al, 29 were from Asia but the detailed geographic origin of these participants was not provided [2]. Finally, the study by Ho et al focused on EUS practice and training in Asia [3]. This study included only those who were practicing endosonographers.

The study by Das et al identified subjects by selecting U.S. and international endosonography registrants at the $13^{\text {th }}$ international EUS symposium [4]. In doing so, that study selected subjects more likely to have knowledge of EUS since they actually practiced the procedure. As a result, that study is less able to provide insight into the general EUS knowledge amongst gastroenterologists in different parts of the world but was aimed more at surveying clinical EUS practice at that particular time point.

Previous studies have shown that gastroenterologists who perform EUS do better in the knowledge assessment regarding EUS compared to non-endosonographers [2]. In the present study, the number of gastroenterologists performing EUS was too small to make a meaningful comparable assessment. Previous studies also show greater knowledge deficits of colorectal indications for 


\section{EUS [2]. We did not find such a difference.}

A limitation of this study is that not all countries in the region are represented, due primarily to their nonparticipation in the conference where the study was conducted. Previous surveys of EUS knowledge have been conducted by email or internet-based survey systems and have been limited by low response rates $[2,4]$. In our study, the questionnaire was handed out physically to participants at a meeting and therefore a $100 \%$ response rate could be achieved. However, because only attendees of this particular meeting were included in the study, a selection bias could have occurred.

The most novel aspect of this study was the investigation into reasons for the lack of widespread EUS adoption in the region. Participants were asked about the barriers or obstacles they perceived impaired EUS in their countries. The obstacles identified includes issues relating to equipment availability, procedure cost, and the lack of trained endosonographers in the region.

In summary, this study provides valuable insight into EUS-related knowledge in a previously unstudied part of the world. The advancement of endoscopic ultrasonography in South and South East Asia requires training and education of
Gastroenterologists in the region and access to EUS equipment and trained practitioners. Gastroenterologists in the region need to educate and train themselves and their trainees in the practice endosonography. International endosonographers and gastroenterology societies could also play a positive role in this neglected region.

\section{References}

1. Dumonceau J-M, Polkowski M, Larghi A, Vilmann P, Giovannini M, Frossard J-L, et al. Indications, results, and clinical impact of endoscopic ultrasound (EUS)-guided sampling in gastroenterology: European Society of Gastrointestinal Endoscopy (ESGE) Clinical Guideline. Endoscopy. 2011;43:21842456. doi:10.1055/s-0030-1256754.

2. Yusuf TE, Harewood GC, Clain JE, Levy MJ. International survey of knowledge of indications for EUS. Gastrointest Endosc. 2006;63:16377326. doi:10.1016/j.gie.2005.09.032.

3. Ho KY. Survey of endoscopic ultrasonographic practice and training in the Asia-Pacific region. J Gastroenterol Hepatol. 2006;21:16872302. doi:10.1111/j.1440-1746.2006.04254.x.

4. Das A, Mourad W, Lightdale CJ, Sivak MV, Chak A. An international survey of the clinical practice of EUS. Gastrointest Endosc. 2004;60:15557952. 